The American Psychological Foundation's (APF's) competitive grant and scholarship program supports the development of psychological talent and mobilizes psychology's resources toward those problems that need to be addressed yet would otherwise go unfunded. Giving to APF supports students and psychologists whose research, projects, and intervention programs help individuals and communities worldwide, making a difference every day.

APF would like to thank the following donors who contributed to the Foundation in 2008:

\section{$\$ 100,000$ and above}

American Psychological

Association (APA)

The estate of Jacquelin R. Goldman

David and Carol Myers

\section{$\$ 25,000$ to $\$ 49,999$}

Theodore and Renee Millon

Asher R. Pacht

Celest Pearsall

The estate of Joanne Sztogryn

Steven Ungerleider

\section{$\$ 10,000$ to $\$ 24,999$}

Barry S. Anton

APA Insurance Trust

Charles L. Brewer

Morris Goodman

Harry and Miriam Levinson

Pearson, Inc.

Andrew A. Shiva and Mrs. Shiva

Turrell Fund

W. Bruce Walsh

Diane J. Willis

\section{$\$ 5,000$ to $\$ 9,999$}

Anonymous

Dorothy W. Cantor

George A. Dunn Jr.

Barbara E. Golden

Carolyn and Stephen Schroeder
Raymond A. and Rosalee G. Weiss

\section{$\$ 1,000$ to $\$ 4,999$}

Patricia A. Bresky

Ronald T. Brown

Mathilda B. Canter

Jan L. Culbertson

Marilyn T. Erickson

Lorraine Eyde

Edwin A. and Pauline S.

Fleishman

Kurt F. Geisinger and Janet F. Carlson

Irving and Carol Gottesman

Elaine F. Greene

Lisa Grossman

Edith H. Grotberg

Helen Grusd

Guilford Publications, Inc.

Douglas C. Haldeman

Joseph H. Hineman

William C. Howell

Gerald P. and Robin C. Koocher

Ronald F. Levant

Ruth G. and Joseph D. Matarazzo

Janet and Lee Matthews

Gary B. Melton

Dolores O. Morris

Pro-Ed, Inc.

James Campbell Quick and Sheri Quick

Carole A. Rayburn

Donald K. Routh

Morgan T. Sammons

Helene Sands

Sylvia Shellenberger

Sandra L. Shullman

Mason A. Sommers

Jeffrey H. Spector

Taylor and Francis Group, LLC

Louise O. Underwood

Kristi Sands Van Sickle

Melba J. T. Vasquez

Wayne Weiten

Kan Zhang

Philip G. Zimbardo and Christina

Maslach

Donald G. Zytowski

\section{$\$ 500$ to $\$ 999$}

Nancy L. Baker

G. Andrew Benjamin

Barbara L. Bonner

Rosalind Dorlen

G. Rita Dudley-Grant

Ronald E. Fox

Craig and Heather Gruber
Harcourt, Inc.

Janet Hodde-Vargas and Luis A. Vargas

Frances Degen Horowitz

Jane Knitzer

Luciano L'Abate

Bonnie Markham

Nancy G. Moore

Herbert C. Quay and Anne E. Hogan

Susan L. Rosenthal

Karen J. Saywitz

Society of Counseling Psychology (APA Division 17)

Gilbert S. Spitzer

Irving B. Weiner

Molly R. Witten

Abraham W. Wolf

Carol Falender Zohn

\section{$\$ 100$ to $\$ 499$}

Ronald and Anna Abeles

Edward G. Abelson

Richard R. Abidin

Daniel J. Abrahamson

Alpert Medical School of Brown

University—Psych Training

APA Membership Board

Association of Psychologists in

Academic Health Centers

Asuncion Austria

Jeffrey H. Axelbank

Gary A. Baffa

Lynn Bailey and J. H. Z. Greiner

Ellen K. Baker

Laura H. Barbanel

Jeffrey E. Barnett

Robert P. Barrell

Stanley Berent

Sharon Berry

Mae Lee Billet-Ziskin

Rita R. Blau

Kenneth H. Bohm

Lawrence J. Bookbinder

Walter C. Borman

Debra Borys

George J. Bouklas

Anne E. Bowes

Thomas E. Bratter

Jeri Breiner

Robert D. Bretz Jr.

Morton D. Brooks

Robert A. Brown

Brown University

Jerome Bruner

Merry Bullock
Anthony A. Calabrese

Angela E. Cass-Prost

Armand R. Cerbone

Alice F. Chang

Marylou Cheal

Lillian Comas-Díaz

David R. Cox

Pamela H. Crabtree

Areta H. Crowell

Walter F. Daves

Henry P. and Tema David

Mitzi J. Dearborn

Michael A. Deem

Irene M. Deitch

Gwendolyn Dekay

Patrick H. DeLeon

Thomas J. DeMaio

Victor H. Denenberg

Florence L. Denmark and Robert

W. Wesner

Constance P. Dent

Alba De Simone

Irma R. De Torres

Theodore Donaldson

Emanuel Donchin

Molly W. Donovan

Marjy N. Ehmer

Thomas D. Elkin

T. David Elkin

Henry C. and Florence P. Ellis

Alan D. Entin

Simon B. Feder

Susanna Feder

Algirdas Fedoravicius

Deborah Felder

Jerry and Joan Ferguson

Lucy R. Ferguson

Seymour and Norma D. Feshbach

Barbara H. Fiese

Elsie Fischer

Eileen F. Fitzgerald

James L. Fosshage

Kenneth A. Frank

Arthur M. Freedman

Howard J. Friedman

Daniel W. Fullmer

Michael R. Furlong

Norman and Edith Garmezy

Kenneth W. Gates

Gerald Gelber

Douglas B. Golden

Clarann J. Goldring

Amy E. Goldstein

Carol D. Goodheart

Gail S. Goodman 
Paula M. Gorlitz

Albert E. Goss

Gloria B. Gottsegen

Harrison Gough and Mrs. Gough

Daniel M. Graffeo

Luise A. Gray

Warren A. Graybiel

Grace S. Green

Rena M. Greenblatt

Richard L. Greenblatt

Kathleen N. Griffin

Seymour Z. Gross

Marvin E. Grunzke

Paul A. Guido

Lee Gurel

Joseph R. Guydish

Carolyn R. Hally

Raymond F. Hanbury Jr.

Robert J. Hartke

Kate F. Hays

Thomas E. Heinzen

Margaret B. Heldring

Marvin Hersko

James D. and Angela O. Herzog

Leslie H. Hicks

Bill Hill

Jane K. Hochberg

L. Richard Hoffman

Jonathan C. Hoistad

Charles H. Holland

John F. House

Ann Howard

Leo G. Hruska

Jerry B. Hutton

John F. Ireland

Anthony Jackson

Louis P. James

James J. Jenkins

Ronald J. Jereb

Suzanne Bennett Johnson

Gary E. Jusela

Jerome Kagan

Gloria Batkin Kahn

Carole A. Kant

Florence Kaslow

Charles D. Katz

Toby B. Kaufman

Jean Cole Kelleher

Herbert C. Kelman

Janice M. Kenny

John J. Kenny

Kenneth A. Klauck

Elizabeth A. Klonoff

Nathan Kogan

Elizabeth A. Kolin

Loretta Kroin

Jean E. LaCrosse

John M. Lagos

Rebecca M. Leblanc

David M. Lechuga

Gregory K. Lehne

Marvin Leibowitz

Robin Leong and Mrs. Leong

Suzanne LeSure

Raymond G. Leugers

Alexander H. Levi

Edwin I. Levy
Neal E. Leynor

Zanvel A. Liff

Marc I. Lipkus

Leland H. Lipp

Antolin M. Llorente

Paul J. Lloyd

R. Duncan Luce

John R. Lutz

William Lysak

Avi Madan-Swain and James Swain

Carol C. Malone

Wendy B. Marlowe

Neil A. Massoth

Margaret W. Matlin

Claudia M. McConnell

Wilbert J. and Virginia

McKeachie

James M. McMahon

Kathleen M. McNamara

Anne B. Menotti

Peter Francis Merenda

Stanley B. Messer

William J. Meyer

Thaddeus Midas

Gilbert and Joan Mintz

Leonard L. Mitnick

Donna D. and Henry H. Morgan

John A. Munoz

John P. Murray

Harold R. Musiker

Lynne Namka

James T. Napolitan

Bernard F. Natelson

Edith D. Neimark

Paul D. Nelson

Gilbert H. Newman

Edmund J. Nightingale

Robert B. Noll

Fay-Tyler M. Norton

Ruth Ochroch

Robert J. Ogg

John F. O'Leary

J. Bruce Overmier

Oxford University Press

Ruth and Albert Paige

Hagop S. Pambookian

James L. Pate

Herbert L. Pick

William S. Pollack

Natalie Porter

Sanford Portnoy

Edith S. Quevedo

Morton H. Rabin

Donna Rasin-Waters

Anthony E. Reading

Elisabeth A. Reading

Jill N. Reich

Mary E. Reuder

Leo J. Reyna

Michael Clark Roberts

Laurie K. Robinson

Jeanine A. Roose

Harold and Linda Rosenson

Edythe Rosenthal

Richard H. Rubes
Nancy Felipe Russo

Ralph M. Rust

David Sacks

William R. Safarjan

O. J. Sahler

David J. Schroeder

Bert and Ruth Schwartz

Thomas B. Scott

James W. Selgas

Virginia C. Shipman

Alex M. Siegel

Dea C. Silbertrust

Reuben J. and Mary M. Silver

Jerome L. Singer

Nancy S. Small

Harrison M. Smith

M. Brewster Smith and Deborah Smith

Michael C. Smith

Ruth E. Smith

Anita O. Solomon

Martha Sue G. Stamper

Trisha A. Stark

Marion P. Steininger

Robert J. Sternberg

Elisabeth R. Straus

Richard C. Sullivan

James F. Sunbury

James W. Thompson

Carole L. Thorsell

Roberta and Sheldon Toll

Henry Tomes

Harry Triandis and Mrs. Triandis

Barbara A. Van Horne

McCay and Marie Vernon

Gary Walco and Jayne Shackter Walco

Robert S. Waldrop

William F. Walsh

Deborah E. Weinstock-Savoy

Robin B. Welch

James G. Wellborn

Joan M. Weltzien

Eloise Werlin

Michael Wertheimer

Susan K. Whitbourne

Susan L. Williams

Anne H. Wright

Lawrence S. Wrightsman Jr.

Ronald D. Wynne

Barbara W. Yee

Robert I. and Gloria Yufit

Jeffrey M. Zacks

Gerald H. Zuk

\section{Less than $\$ 100$}

Ira E. Aaron

Rosemary Adam-Terem

Harriet Ainbinder

Rafic Ali Al-Banawi

Lynda Albert

David G. Alden

Mary E. Alexander

Julie A. Allender

David A. Altfeld
American Board of Professional Psychology

Sue Anita Ammen

Joan S. Anderson

Alexander Antwarg

Helen P. Appleton

John R. Araza

Mark D. Attridge

Robert A. Auger

Elaine Axelman

Teodoro Ayllon

David H. Backus II

Suzanne Baker

Sarah J. Banks

Walter B. Barbe

Jacob L. Barber

Linda F. Barnhurst

Edmund S. Bartlett

Ellen K. Bass

Brian R. Bate

Lucinda M. Beard

Muriel Y. Beattie

Sharon L. Ben-Meir

Karen S. Berkowitz

Jane H. Bernstein

Paula P. Bernstein

Standley L. Bivens

Edward Blacker

Leonard S. Blackman

Andrew D. Blair

Richard G. Blouch

Stanley and June E. Blum

Lynn K. Boatwright

Richard R. Bocchin

Judith M. Body

Joseph A. Borello

Bruce G. Borkosky

Linda L. Bortell

Bette L. Bottoms

Laura Boyd

Malcolm K. Brachman Jr.

Georgia W. Brandstadter-Palmer

Beverly A. Brauer

A. Richard Brayer

Sharon Brennan

Thomas P. Brennan

Belinda P. Brent

Inge Bretherton

Mary S. Brizzolara

Jane P. Brooks

Idalyn S. Brown

William E. Brown

Martha J. Brownlee-Duffeck

Charles S. Brudo

Sharon B. Buchbinder

Nancy G. Burgoyne

Robert John Burns

Katherine Burrows

Charles E. Burt

James E. Byassee

Marilyn S. Cabay

Peter Calapai

Robert C. Calfee

Bonnie W. Camp

Arthur Canter

Maury T. Carlin

Dorothy T. Carlson

Annalise L. Caron

Janeth T. Carpenter 
Linda Carter

Martin P. Chaplin

Edwina P. Chappell

Hermine J. Chern

Sasha Chernoff

Gloria T. Chisum

Deanna G. Chitayat

Salvatore Cianci

Walter J. Ciecko

Pauline A. Clansy

Barbara L. Claster

Daniel S. Claster

Alexander Cohen

Allan Y. Cohen and Elana U. Cohen

Karen S. Cohen

Murray L. Cohen

Robert C. Colligan

Kenneth G. Cook

Sherri Coomer

Dewey G. Cornell

Carol C. Cossum

Mary E. Courtney

Mary L. Cox

Bruce E. Crapuchettes

Bruce E. Crow

Richard D. Cummings

G. Scott Curtis

J. Allison Curtis

Norvin R. Curtis

Susan C. Czernicka

Samuel L. Dambrocia

Leila F. Dane

Puspa M. Das

J. Sanford Davis

Michael L. Davis

William E. Davis

Steven C. DeAlmeida

Michael T. Dealy

Eric L. Deitchman

Ann L. De Lancey

James W. Delaney

Cynthia De Las Fuentes

H. Brent Dietsche

Stephen C. Dijulio

Gena M. Dilabio

Division of Psychotherapy (APA Division 29)

W. Robert Dixon

Alan L. and Linda A. Doerman

Nancy N. Doi

Arthur A. Dole

Steven H. Domann

Alan C. Domian

George Donoian

Guerson Doricent

Edward J. Dougherty

John C. Dovel

Susan Downing

Charles A. Drewes

Joseph E. Driess

Sari H. Dworkin

Carol J. Eagle

Paul A. Eckert

Gerald Edelstein

Manuel H. Edquist

Laura A. Eisdorfer

Marlene R. Eisen

Gilbert J. Eisenberg

Katie Eisenstadt
Phoebe C. Ellsworth

Robert A. Embree

John Engler

Virginia Enrico

William M. Erwin

Alexander Eschbach

Garrett B. Essres

April E. Fallon

Angela S. Fanelli

Norman L. Farberow

Anne R. Farrar-Anton

Paul Fedio

Steven Feldgaier

Michael Feldman

Michael A. Fenichel

Robert B. Field

Joan C. Fiorello

Paula B. Firestone

Mark A. Fish

Gerald Fleischer

Mark Flescher

John D. Fletcher

J. M. Fontinell

Mary S. Foote

Linda M. Forrest

Jane L. Forsyth

John R. Fowler

Christopher Fox

Paul D. Frederickson

Antonia C. Fried

Abby Lynn Friedman

Monroe P. Friedman

Thomas P. Frio

Michael J. Fulop

Joseph Ganz

Kerry L. Garretson

John E. Garrison

Caesar T. Gaza

Dennis J. Geiger

Jacquelyn H. Gentry

David A. Gershaw

Evelyn L. Gerson

Shelley L. Gerson

Irene B. Giessl

Carlyle W. Gilbertson

William Gingold

Efrat Ginot

Macario Giraldo

Alan J. Glasser

Deborah W. Gleaves

Alyce M. Gligor

John P. Godfrey

Katherine G. Godsman

Arnold G. Gofstein

Shelley Goldklank

Diana S. Goldstein

Maraika Gooding

Debra E. Gordon

Jack Gorelick

Dennis A. Gorman

Adele E. Gottfried

Allen W. Gottfried

Joel E. Grace

Cynthia M. Gracianette

John L. Grahm

Anita M. Gram

Arthur A. Gray

Joann H. Grayson

Caesar C. Gregory

Brenda Q. Gretzinger
Herbert W. Gross

Jules Grosswald

John J. Gualtieri

Maria J. Guglielmino

Robert L. Haley

Cheryl L. Hall

Robert M. Hamm

Rodney W. Hammond

Jo-Ida C. Hansen

Robert A. Hanson

James H. Harding

Norma J. Hart

Patricia Harteneck

John G. Hartung

Stefani L. Hathaway

Judith M. Haupt

Robert P. Hawkins

Mary J. Hayden

Norman D. Henderson

Robert D. Hendricks

Winifred Hentschel

Susan J. Herman

Gerald H. Hermanson

William C. Heusler

Jo M. Heyman

Karen and J. Gary Hickman

Robert A. Hicks

Marjorie J. Hill

William A. Hillix

David L. Hoats

David A. Hoffman

Diana W. Hoffman

Sandra S. Hoffman

David K. Hogberg

Stephen L. Holliday

Susan Burchfield Holliday

Thomas G. Hollis

Joseph H. Honor

Hyman Hops

Richard P. Horevitz

Carrell P. Horton

Robert A. Horwitz

Robert R. Houghton

William C. House

Linda S. Huganir

Diane J. Huisinga

Irene M. Hulicka

Scott J. Hunter

Jill E. Hunter-Williams

Barry A. Hurwitz

Arnold Hyman

Cathy K. Iger

Monica J. Indart

Fred H. Ireland

Emily S. Israel

Robert M. Itatani

Dennis J. Jacobs

Sheryl R. Jacobs

Marion Jahelka

Kay Jamison

Dolores J. Janecky

David G. Jarmon

Michael Jarrett

Lawrence G. Jarvis

Rade R. Jenkins

Robert N. Johansen

Deborah H. Johnson

Marcia K. Johnson
William G. Johnson

Clifford G. Jordan

Paul P. Kadis

Michael Kahn

Thomas C. Kalin

Lisa A. Kaneshiro

Julie P. Kantor

Audra H. Kaplan

Ralph V. Kaplan

Julie A. Karp

Martha A. Karson

Roberta Katz

Charles C. Keach

M. Jean Keeley

Lucille Keenan

Sally A. Keller

John D. Kelton

Edward J. Kelty

Miriam F. Kelty

James I. Kepner

Ilene M. Kesselhaut

Marta F. Ketchel

Irwin W. Kidorf

M. Marlyne Kilbey

Sally J. Kilmer

JungSook Kim

M. Elizabeth Kingsley

Emily B. Kirby

R. Vernon Kirk

Neil M. Kirschner

Ronan M. Kisch

Chaeleyah K. Klein

Steven Klein

Raymond A. Knight

Judith S. Goldberg Kolman

Edward J. Korber

Jeanne M. Kotuby

Judith A. Kramer

Leonard F. Krause

Iseli K. Krauss

John H. and Bonnie Krystal

Anthony M. Kuchan

Suzanne Kunkle

Stephen A. Kushnick

Vesna Kutlesic

Frances La Barre

Linda D. Ladd

Phyllis R. Lakin

Kiumars Lalezarzadeh

Peter J. Lamb

William K. Lamb

Jay M. Land

Matthew G. Lanna

Steven M. Lee

Gerald D. Leibowitz

Irene W. Leigh

Martin E. Lemon

Irving G. Leon

Diane Lessner

Judith C. Levey

Samuel Levinson

Julie M. Levitt

Theodore C. Lewandowski

Selma Lewis

Kenneth R. Liberatore

Harvey J. Lieberman

Brian M. Lippincott

Barbara J. Lips

Lewis and Edna Lipsitt

Nechama Liss-Levinson 
David T. London

George J. Lordi

Deirdre V. Lovecky

Faye Lovrinic

Marc I. Lubin

D. Marie Lucente

Marianna Luck

Elizabeth Magnus

Yash P. Manchanda

Donald W. Manthei

Brian A. March

Jane L. Marcus

Nathaniel S. Marcus

Thomas E. Martin

Wilfred W. Martin

Ann L. Martinko

Susan Mathes

Catherine B. Maxwell

Donald C. McCann

Kellie M. McCants-Price

George O. McClary

Honor E. McClellan

Mercedes McCormick

Laura M. McCrary Hansen

McDonald, Roth, \& Associates, PA

Christine McElroy

John F. McGowan

David J. McIntyre

M. C. McKinley

Romella J. McNeil

Paul W. McReynolds

Patricia H. McVeigh

David P. Meshorer

Leonard J. Meyerson

Steven R. Michelson

David F. Middleton

Judith-Annette Milburn

Diana L. Miles

John G. Miller

Katherine J. Miller

Patricia Miller

Martin M. Mintz

Jeffery S. Mio

Charles D. Miron

Alicia M. Mitchell

Peter N. Moore

Carole W. Morgan

Denise N. Morita

Patricia Morris

Catherine G. Morrison

Margaret E. Richards Mosher

John F. Mulkeen

John J. Munk

J. A. Munoz

Debra A. Murray

Morgan J. Murray Jr.

Richard J. Muszynski

Edward M. Mwelwa

Ernest R. Myers

Gilead Nachmani

Matthew L. Nance

Sophie Narayan

John M. Nash

Gene K. Nebel

Franklin and D. Joan Neff

William H. Nelson

Edmund C. and Olga M. Neuhaus

Slater E. and Patricia T. Newman

Edward R. Nishball
Camille M. Norvell

Stanley Novak

Nancy L. Nussbaum

J. Brien O'Callaghan

John A. Ohlson

Eric J. Oritt

Miriam R. Osofsky

Dawn A. Osterweil

Jan Owens-Lane

Art Pacheco

Laura K. Palmer

Ethel and Jacques Pankove

Karen D. Pape

Paul D. Park

Yetta Parker

Thomas S. Parsons

Jeannie V. Pasacreta

George E. Passey

L. T. Payne

Harvey Pearl

Martha Pearse

Carol Pepper

Hilda D. Perlitsh

Evelyn B. Perlman

Mark W. Perrenoud

Louis A. Perrott

Shirley E. Peters

William D. Petok

Michael J. Philipp

Gabriella K. Philippou

Barbara E. Phillips

Julia Phillips

James W. Pier

Rolffs S. Pinkerton

Geraldine K. Piorkowski

Barbara Pitcher

William D. Pithers

Willis D. Poland

James R. Pomerantz

Edna S. Popoff

Gayle K. Porter

Marion B. Powell

Fawn Powers

Laura Primakoff

Anne and Louis Primavera

Paul W. Proctor

Diane J. Prosser

Richard Quaglino

James P. Quinlan

Franklin D. Raddock

Lisa E. Rafkin-Mervis

Jenny Rajput

Idalia Ramos and Steven D. Hurlburt

Jacqueline L. Rapier

Elliot J. Rapoport

Teresa A. Rattray

John J. Rearden

Martin and Enid F. Reiser

Jaquelyn L. Resnick

Anne W. Reynolds

Nelson C. Ribble

David C. Riccio

Alan R. Rickfelder

Lawrence G. Ritt

Ellen Rittenberg

Debra C. Roach

John D. Robinson
David C. Rodeheffer

Elaine Rodino

James G. Rogers

Linda Ronald

Edward Ronstadt-Martinez

Helen Rose

Sandra S. Rosenblatt

Judy F. Rosenblith

Paul I. Rosenthal

Ronald L. Rosenthal

Mark R. Rosenzweig

Charles Rothstein

Daniel L. and Grace Rothstein

Phyllis B. Rubin

Thomas M. Ruby

Lawrence Rudin

Sandra W. Russ

Judith S. Ruzumna

Karen A. Ryabchenko

Carolyn S. Ryan

Renate K. Safrin

Michael J. Salamon

Carole A. Salvador

Gilbert O. Sanders

Gary M. Sater

Wendy Satin-Rapaport

Andrew Savicky

Bianca M. Schaefer

Rodney R. Schall

Jerry S. Schecter

Linda W. Scheffler

Paul W. Schenk

Milo W. Scherer

Peter H. Schild

Tammera A. Schmalz

Laura S. Schnaps

Phyllis Schottenstein

Jerry D. Schrum

Martin G. Schuh

Celia L. Schulhoff

Melvin S. Schwager

Jean Schwartz

Lita L. Schwartz

Sheryn T. Scott

Elizabeth Seebach

Jack Segal

Richard J. Seime

Gerald T. Semmler

Jeffrey G. Sharp

Juanita Shell

Robert L. Shellenberger

Reginald R. Shepps

Kari Sherman

Nissim Shimoni

Martin M. Shinedling

Suanne Shocket

Sandra L. Shulmire

Alan B. Siegel

Linda Silber

Herman R. Silbiger

Judith L. Silverstein

Dale D. Simmons

Dorothy G. Singer

Michael A. Sitar

Jacob E. Slutzky

Carole P. Smith

Roberta A. Smith

Stanley J. Smits

May Soll

Irwin and Elizabeth Sollinger

Sondra E. Solomon
Philip H. Sorensen

Alphonso L. Sorhaindo

Michael B. Sperling

Violet E. Spraings

Jennifer Spring

Springer-SBM, LLC

Walter C. Stanley

Sheree E. Starr

John A. Stern

Vaughn E. Stimbert

Anne H. Strohm

Mary O. Styrt

Judith A. Sugar

Daniel A. Sugarman

Brian W. Sugden

Bradley D. Sussner

Daisy K. Switzer

Tami T. Swonigan

James M. Sydnor-Greenberg

Barbara A. Sziraki

Akenori Takeda

Harold Takooshian

Virginia Tams

Aldo J. Tartaglini Jr.

James A. Taylor

Carl E. Thomas

Robert J. Thomas Jr.

Curtis E. Thomsen

Daniel G. Thomsen

Carol W. Tierney

Carol K. Tittle

Alicia M. Todd

Laura C. Toomey

Mario A. Torres

Michael R. Tramonte

Ping Chung R. Tseng

Mitchel B. Turbin

Terry G. Vavra

Sandra L. Vedovato

Guillermo Velazquez-Flores

Roberta B. Vogel

David S. Wachtel

Janet S. Waksman

Raymond O. Waldkoetter

Martha W. Waller

Montira L. Warran

Stephanie Warren

Janet C. Waterhouse

Mitchell L. Wax

Debra G. Wayne

Trevin D. Wear

Margaret L. Weber-Levine

Kenneth H. Webster

Florence Wechsberg

Sara C. Wedeman

Constance Weil

S. Dana Weinberg

Linda L. Weinhold

Charles D. Weinstein

Andrea Weiss

Glenna Weiss

Janet M. Weiss

Elizabeth Welker

Donald R. Welti

Miriam J. Wendt

Stephen A. Werbel

Calvin and Elizabeth Wettstein

Michael Wexler

Joffre T. Whisenton 
Earl M. White

Jacquelyn W. White

J. K. Whitt

Harley R. Wideman

Donald L. Wiedis

Jeffrey R. Wilbert

Walter L. Williams

Georgene R. Winick

Marrea A. Winnega

Myra J. Wise

Bonnie Wolf

Lisa H. Wolfe

Larry J. Wornian

Massi H. Wyatt

Thomas P. Zahn

Michael J. Zborowski

Sherry L. Zemlick

Ellen D. Zide

\section{Legacy Club} Members

Norman B. Anderson

Anonymous

Lyle Eugene Bourne

Charles L. Brewer

Laura Brown

Alex Carballo-Dieguez

Alice F. Chang

Helen L. Coons

Nicholas and Dorothy Cummings

Henry P. and Tema S. David

G. Rita Dudley-Grant

Marilyn T. Erickson

Oliva M. Espin

Linda Forrest

Raymond and Sandra Fowler
Morris Goodman

Stanley Graham

Lee Gurel

Frances Degen Horowitz

Ann Howard

Steven E. James

Jean Cole Kelleher

Douglas Kimmel

Harry and Miriam Levinson

Robert A. and Phyllis Levitt

J. Nina Lieberman

Lewis P. and Edna Duchin Lipsitt

Rodney Lowman and Linda

Richardson

Elaine Lundahl

Ruth G. and Joseph D. Matarazzo

Wilbert McKeachie

Roberta Meier

Arlyn H. Miller
Edmund Nightingale

John Noon

Mary Ellen Olbrisch

James Campbell Quick and Sheri Quick

Annette U. Rickel

Morgan Sammons

Ruth and Jerry Seitler

Martin E. P. Seligman

Patricia A. Smith

Charles D. and Carol Spielberger

Franklyn Springfield

George and Joan Stricker

Raymond A. and Rosalee G.

Weiss

William J. Woods 